\title{
Innovación en la contratación de una EDM. El caso de Pamplona.
}

\author{
José Luis Díaz Carrero \\ Técnico de Planificación de la Mancomunidad de la Comarca de Pamplona, España \\ María Moral Gurrea \\ Letrada de la Mancomunidad de la Comarca de Pamplona, España
}

Jesús Velasco Pérez

Director del Área de Transporte de la Mancomunidad de la Comarca de Pamplona, España

\section{RESUMEN}

La Mancomunidad de la Comarca de Pamplona es la autoridad del transporte de la aglomeración urbana de Pamplona. Dentro de sus cometidos, figuran los relativos a la gestión y planificación de la red de transporte urbano comarcal.

En enero de 2013 se establecieron las bases de la Encuesta Domiciliaria de Movilidad (EDM 2013). Se dividió el proyecto en cinco contrataciones diferentes que interrelacionadas entre ellas, configuraron el trabajo final que desembocó en una propuesta de una nueva red de transporte basada en ejes troncales de alta capacidad (BRT).

La primera fase consistió en un trabajo previo para establecer la zonificación y así dimensionar adecuadamente el trabajo de campo de la encuesta domiciliaria.

La segunda fase se centró en la selección del equipo consultor que llevaría el grueso de los trabajos, incluyendo las determinaciones técnicas de las campañas de campo posteriores.

La tercera fase (tres últimas contrataciones) referidas al trabajo de campo, se encargaron del grueso de la encuesta domiciliaria, de los parámetros del modo autobús y de una campaña de preferencias declaradas para caracterizar los modelos de demanda en relación con el transbordo y aparcamiento.

Los trabajos se realizaron bajo la premisa de no hacer un fraccionamiento del contrato sino con el principio de conseguir una mayor especialización de los trabajos.

La principal ventaja obtenida de este sistema ha sido la calidad final obtenida en los trabajos a pesar de la elevada carga de trabajo administrativo que ha sido necesario acometer.

\section{LA MANCOMUNIDAD DE LA COMARCA DE PAMPLONA}


La Mancomunidad de la Comarca de Pamplona es una entidad local que presta los servicios del ciclo integral del agua, residuos urbanos, transporte urbano comarcal, taxi y parque fluvial de la comarca. Estos servicios se prestan a una población de 361.402 habitantes de 50 municipios.

En junio de 1998 se aprueba la Ley Foral 8/1998 del transporte regular de viajeros de la Comarca de Pamplona que prevé como solución de integración administrativa una autoridad común de transporte para la que propone una administración ya existente e integrando en la actualidad a 18 de los 50 municipios que integran la Mancomunidad de la Comarca de Pamplona. En total, conforma un área metropolitana de 339.128 habitantes.

\subsection{Pasos previos}

La Mancomunidad de la Comarca de Pamplona (MCP), ejerciendo las competencias que le asigna la Ley Foral 8/1998 de 1 de Junio relativas a la planificación y a la inspección de los servicios de transporte, realiza actuaciones encaminadas a la mejora y optimización del sistema de Transporte Urbano Comarcal (TUC) en los 18 municipios de la Comarca de Pamplona que han delegado sus competencias de transporte.

Para ello, se proponía realizar un estudio de reordenación de la red del TUC, mediante la utilización de herramientas de modelización de transporte para sentar las bases de las futuras actuaciones a corto y medio plazo.

El anterior estudio similar, se realizó en 2002 ("Estudio de reordenación de la Red de Transporte Urbano Comarcal”, Mancomunidad de la Comarca de Pamplona 2002).

La última encuesta domiciliaria de Movilidad se realizó en 1996 por el Gobierno de Navarra. Posteriormente el Ayuntamiento de Pamplona realizó una encuesta domiciliaria en 2004, con una posterior reconstrucción del ámbito para llegar a la Comarca completa (Gobierno de Navarra, 2006).

\section{CONTRATACIONES}

Desde un punto de vista de la gestión del proyecto encomendado, se pretendía segregar trabajo de campo del trabajo de gabinete. El objetivo fundamental de esta segregación era doble: garantizar un buen trabajo de campo y no ceder presupuesto de campo a trabajo de gabinete.

Inicialmente se pensó en tres contrataciones diferentes;

- Zonificación, para la determinación de los trabajos posteriores y sobre todo, para el volumen de trabajo de campo a realizar. 
- Consultoría, para coordinar los trabajos y generar el control sobre el equipo de campo, del que ya había participado en la determinación técnica del alcance de la contratación y por supuesto, para elaborar el trabajo final.

- Trabajo de Campo de recogida de la información domiciliaria.

Posteriormente, se decidió subdividir el trabajo de campo en tres contrataciones para dotar de agilidad a los equipos ya que la toma de datos se realizaría en poco más de un mes y el esfuerzo en medios humanos podría complicar los trabajos. Finalmente, se ejecutó el trabajo con las cinco contrataciones que se presentan.

\subsection{Contratación de la zonificación}

El objeto de esta fase del trabajo fue la elaboración de una nueva zonificación de transporte, adaptada al contexto metropolitano de 2013 y a las necesidades planteadas de cara a la realización de la encuesta domiciliaria de transporte. Determinar la zonificación se consideró clave, desde un principio, ya que nos permitía definir con mucha mayor precisión los siguientes trabajos, tanto en alcance como en coste; se propuso reducir sensiblemente el número de zonas de encuesta manejadas en anteriores estudios, dejándolas en torno a las 90.

La contratación, al ser inferior a $15.000 €$ se realizó con solicitud de oferta a la Universidad de Navarra, que ya había colaborado en las anteriores zonificaciones de transporte. La duración fue de 6 meses (marzo a septiembre de 2013), estableciendo un pequeños solape con la consultoría para obtener las posibles sugerencias del equipo de consultoría.

\subsection{Contratación de la consultoría}

El objeto del contrato era realizar un estudio de reordenación de la red de Transporte Urbano Comarcal mediante la utilización de herramientas de modelización de transporte y analizando dos tipologías de red de transporte público: "radial” y "mallada".

Adicionalmente, y con carácter previo, se debía realizar la asistencia técnica de los trabajos de Encuesta de Movilidad para la caracterización de un día laborable y lectivo en la Comarca de Pamplona. Las funciones principales en este sentido eran las de colaborar en la definición técnica del pliego técnico para la contratación y dirigir los trabajos de campo que luego servirían para las siguientes fases de definición de la movilidad y de definición de las propuestas de red.

El contrato se licitó por procedimiento abierto inferior al umbral comunitario, de conformidad con lo dispuesto en el artículo 65 de la Ley Foral 6/06 de Contratos Públicos, a la oferta que se considerase más ventajosa, atendiendo a los criterios de adjudicación establecidos en la cláusula 8, sin perjuicio de la facultad de la Mancomunidad de declararlo desierto. El precio de licitación fue de $114.200 €$ para una duración estimada de 15 meses. 
Según una modificación de la LF 6/06 el pliego debería contemplar al menos un 50\% de los puntos de forma objetiva (el licitador conoce al menos el 50\% de los puntos al presentar la oferta).

Se presentaron 9 ofertas técnicas, todas ellas de un nivel razonablemente bueno; en parte debido al filtro de solvencia técnica que se había establecido en el pliego. Una vez realizada la apertura de la oferta económica, se tuvo la excepcionalidad de unas propuestas económicas anormalmente bajas, en el entorno del $50 \%$, que finalmente fueron excluidas.

La adjudicación se produjo a favor de Tema Grupo Consultor con una baja del 33,77\%. Se firmó el contrato el 9 de julio de 2013. El primer trabajo que debía realizar el equipo seleccionado era el de cerrar el pliego técnico con aquellas especificidades técnicas que considerasen para el lanzamiento de la siguiente contratación; el trabajo de encuesta domiciliaria de movilidad.

\subsection{Contratación de la encuesta domiciliaria.}

El objeto de esta contratación era la caracterización de la movilidad general en la Comarca de Pamplona en un día laborable y lectivo. Debería recoger las pautas de movilidad de todos los modos de desplazamiento: vehículo privado, transporte público, a pie, taxi, moto, bicicleta, etc.

Se debían por tanto realizar tres trabajos dentro de la campaña de campo:

- Encuesta Domiciliaria de Movilidad

- Encuesta de Movilidad en Centros Atractores

- Encuestas Pantalla al Vehículo Privado.

Las campañas se licitaron en base a la conjunción de los precios unitarios y las muestras mínimas a obtener. En el caso de la Encuesta Domiciliaria de Movilidad el coste unitario correspondió al coste de cada encuesta completa al hogar (incluye cuestionario del hogar y las encuestas a cada uno de los residentes en ese domicilio), mientras que en el caso de las otras dos investigaciones, la Encuesta en Centros Atractores y las Encuestas Pantalla, el coste unitario se correspondería al coste de horas de encuestador y/o aforadores.

En la Encuesta Domiciliara de Movilidad el número de encuestas domiciliarias a realizar fue de 4.200 y en la Encuesta en Centros Atractores y Encuesta Pantalla el número de horas de encuestas y aforos será de 1.400 horas con el siguiente desglose aproximado:

- Centros Atractores: 15 centros durante 16 horas.

- Aforos Pantalla: 8 puntos durante 16 horas.

- Encuestas Pantalla: 8 puntos durante 12 horas.

El contrato se licitó por procedimiento abierto inferior al umbral comunitario, de 
conformidad con lo dispuesto en el artículo 65 de la Ley Foral 6/06 de Contratos Públicos. Se presentaron 6 ofertas, de las que se excluyeron 4 al no alcanzar la puntuación mínima dentro de la valoración técnica. Finalmente, una vez abierta la oferta económica, se adjudicó a COTESA con un precio unitario 19,80€ por encuesta completada.

La ejecución de los trabajos comenzó a finales de septiembre de 2013 y el trabajo de campo se completó el 29 de noviembre, entregándose todo el material depurado, a los tres meses, a finales de febrero de 2014.

\subsection{Encuestas complementarias.}

Para complementar la medición de la movilidad era necesaria una medición de los modos de transporte para poder realizar la calibración de los modelos y completar la información en relación al transporte público y la velocidad de circulación, que fueron objeto de una nueva contratación.

Los trabajos principales eran la realización de las siguientes campañas de campo:

- Aforo sube/baja y encuesta Autocumplimentada en el Transporte Urbano Comarcal.

- Análisis de la velocidad comercial y las causas de detención en el Transporte Público.

- Análisis de la velocidad de circulación del vehículo privado en el viario.

El contrato se licitó por procedimiento negociado al ser el valor estimado, inferior a $60.000 €$. Se invitó a tres empresas y se realizaron dos rondas de negociación. Siendo adjudicados finalmente a Conchi Rico, que licitó como persona física.

Los trabajos se realizaron la segunda quincena de noviembre, de forma simultánea a la encuesta domiciliaria de movilidad.

\subsection{Preferencias declaradas}

Como contratación final de los trabajos, a la vista de las necesidades del proyecto, como consecuencia de la necesidad de calibrar los modelos, con ciertas variables que se decidió no estimar para conseguir mayor fiabilidad en la modelización, se planteó la necesidad de realizar un trabajo de determinación de los siguientes elementos:

- Captación de las variables que influyen en el transbordo

- Medición de los factores que vinculan aparcamiento de vehículo privado y uso del transporte público

- Medición de los factores que explican el trasvase modal entre vehículo privado, transporte público y peatón.

Estos trabajos se contrataron previa invitación a tres empresas y se adjudicó a la más barata, por un importe de $8.500 €$ ya que el trabajo estaba muy acotado. Lo realizó Eryba en junio 
de 2014, con una duración de dos semanas de trabajo de campo.

\section{CONCLUSIONES}

El proyecto resultó satisfactorio a la vista de los resultados finales obtenidos, aunque quizá la carga de gestión administrativa ha resultado elevada. Se partía de la hipótesis de que cuando se contrata el trabajo de campo de forma separada a la consultoría, no siempre es la mejor combinación ya que exige de una coordinación de los trabajos que es necesario conseguir.

Esta implicación del equipo de consultoría en la determinación del alcance del trabajo de campo se puede conseguir mediante la colaboración en las determinaciones técnicas ya que, posteriormente, deberán basar su trabajo en la calidad de la obtención esos datos.

Las contrataciones se realizaron bajo la premisa de no hacer un fraccionamiento del contrato, sino con el principio de conseguir una mayor especialización de los trabajos.

A tal efecto, si bien todas las contrataciones tenían una finalidad común, el objeto de las mismas era distinto, así como la solvencia técnica exigida para cada uno. Para ello era fundamental la descripción de las necesidades a satisfacer con cada una de las contrataciones, y la definición del objeto del contrato de forma precisa, de tal forma que quedara acreditada la idoneidad del mismo para satisfacer dichas necesidades.

Por otra parte resultaba también decisiva, la determinación del nivel de solvencia técnica exigida para participar en la licitación, partiendo de la necesaria proporcionalidad y adecuación al objeto del contrato que exige la ley.

Todo ello destaca la importancia del conocimiento, desde un puesto de vista técnico, de las necesidades a satisfacer así como de las soluciones que ofrece el mercado, para que finalmente la contratación que se realice cumpla con el alcance del proyecto en su conjunto. 